\title{
Performance Analysis of IP Micro-mobility Handoff Protocols ${ }^{1}$
}

\author{
Chris Blondia ${ }^{1}$, Olga Casals ${ }^{2}$, Peter De Cleyn ${ }^{1}$, and Gert Willems ${ }^{1}$ \\ ${ }^{1}$ University of Antwerp \\ Universiteitsplein 1, B-2610 Antwerp - Belgium \\ \{chris.blondia, peter.decleyn,gert.willems\}@ua.ac.be \\ 2 Polytechnic University of Catalunia \\ Gran Capitan, Mod. D6, E-08071 Barcelona - Spain \\ olga@ac.upc.es
}

\begin{abstract}
Micro-mobility protocols have been proposed to provide seamless local mobility support. This paper focuses on the performance of the handoff schemes of two candidates for micro-mobility protocols, namely HAWAII and Cellular IP. For each handoff scheme, a simple analytical model is developed for the evaluation of two characteristic performance measures: the packet loss probability during handoff and the extra delay experienced by packets that are involved in the handoff. Application of these models allows a comparison of two important handoff schemes: the Multiple Stream Forwarding scheme of HAWAII and the Semi-soft Handoff scheme of Cellular IP.
\end{abstract}

\section{Introduction}

Handheld computing devices, such as palmtop computers are becoming the platform of choice for nowadays personal applications. With the evolution of these devices from a limited communication support, typical point-to-point interfaces (PSTN modem or RS-232 cable), towards high-speed packet radio access interfaces, the demand for network access to mobile users will grow exponentially. The wireless network access infrastructure will have to support a variety of applications and access speeds that should result in a service with the same level of quality as wireline users. Higher speed can be achieved in a cellular network by considering smaller cells. However, the smaller the cells are, the higher the frequency of handoffs may be. A number of access networks use network-dependent solutions for mobility management (e.g. GPRS). In this paper, we focus on Layer 3 solutions only.

Mobile IP [1], the current support of mobility in IP networks, delivers packets to a temporary address assigned to the mobile host at its current point of attachment. This temporary address is communicated to a possibly distant Home Agent. This approach applied to an environment with frequent handoffs may lead to high associated signalling load and unacceptable disturbance to ongoing sessions in terms of handoff latency and packets losses. Therefore, a hierarchical mobility management approach has been proposed (see e.g. [2]), where Mobile IP supports wide area mobility (e.g.

${ }^{1}$ Part of this work was supported by the EU, under project IST 11591, MOEBIUS. 
mobility between different operators) while local mobility is handled by more optimized micro-mobility protocols. These protocols should incorporate a number of important design features related to location management, routing and handoff schemes. They should fulfil requirements such as simplicity to implement, scalability with respect to the induced signalling, efficiency and performance with respect to packet loss and introduced delay. This paper focuses on only one aspect, namely performance evaluation of handoff schemes.

More specifically we evaluate the performance of two prominent solutions for micro-mobility support, namely HAWAII ([3],[4]) and Cellular IP ([5],[6],[7]). For each protocol we develop an analytical model that allows computing characteristic performance measures of the handoff scheme that is used. These measures are related to packet loss and experienced delay. The models that are proposed are simple $\mathrm{M} / \mathrm{M} / 1$ queueing networks that incorporate propagation delays between routers and processing times within routers. The models are not developed for dimensioning purposes, but mainly to investigate the influence of important design parameters and to compare the solutions. For this reason we have assumed Poisson background traffic and exponential processing times. The accuracy of this approximation has been discussed in [8] for the HAWAII protocol. The analytical model has been validated through simulation showing the accuracy of the model. Therefore, a validation is omitted in this paper. The simplicity of the model also allows the study of more general network topologies than the one considered in this paper. Remark that other issues related to these protocols are important (e.g. security) but are out of the scope of this paper.

The remainder of this paper is structured as follows. In Section 2, we present the reference network used to evaluate the handoff schemes. In Section 3, we describe the HAWAII protocol, we present the analytical model and obtain some performance measures. In Section 4we do the same with Cellular IP. In Section 5we compare some performance aspects of the MSF scheme of HAWAII and the Semi-soft Handoff scheme of Cellular IP. Finally Section 6 concludes the paper.

\section{Reference Network}

For the description of the two handoff protocols and their performance evaluation, we will use the following reference network (see Fig. 1). A Mobile Host (MH) moves between two Base Station (Old Base Station BSO and New Base Station BSN). Packets originating from the Corresponding Node $(\mathrm{CN})$ reach BSO (resp. BSN) via the crossover router $\mathrm{R} 0$ and the intermediate router $\mathrm{R} 1$ (resp. R2). We introduce the following notations. Let $\mathrm{Rx}(\mathrm{X})$ denote the random variable defined as the time needed for a packet to be processed by router $\mathrm{Rx}$, leaving via interface $\mathrm{X}$. In other words, it denotes the time between the arrival of a packets at the router and its departure through output interface X. (Same notation applies to "routers" BSO and BSN). Furthermore, let $(\mathrm{Rx}, \mathrm{Ry})$ denote the propagation time on the link between router $\mathrm{Rx}$ and router Ry. 


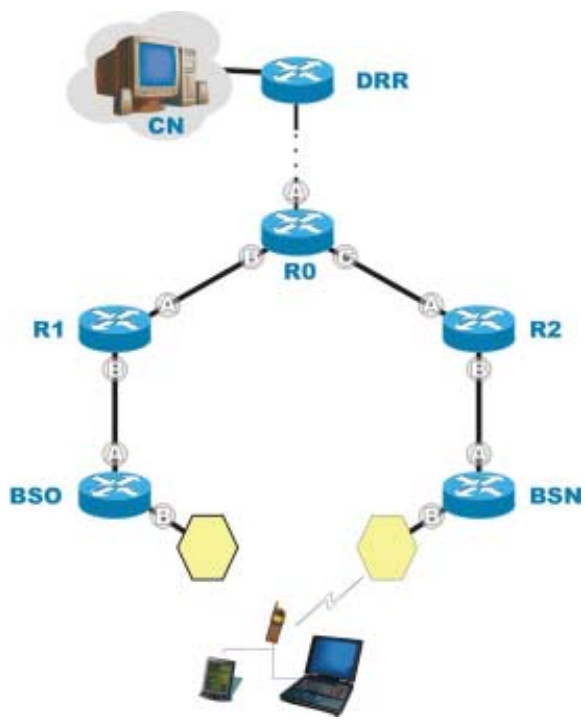

Fig. 1. Reference Network

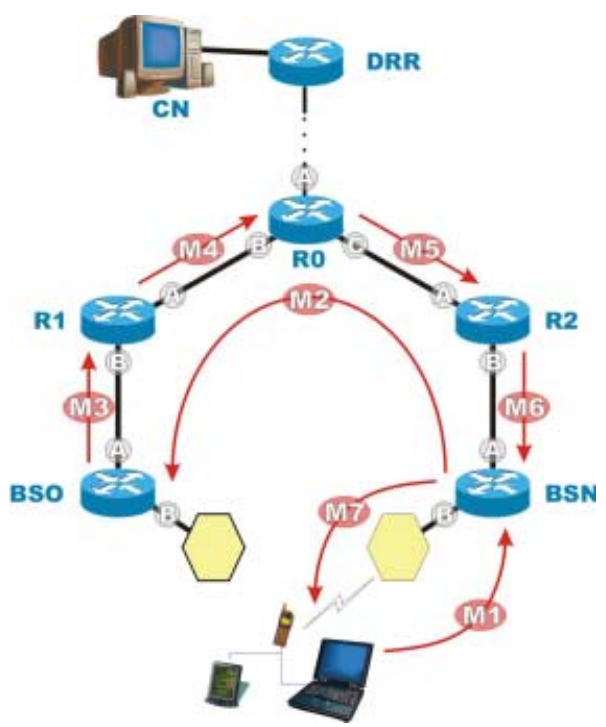

Fig. 2. Messages in HAWAII MSF

\section{HAWAII}

In this Section we describe the handoff mechanism used in HAWAII and present an analytical model to evaluate the packet loss and delay experienced by packets involved in a handoff.

\subsection{Network Model and Routing}

HAWAII is a domain-based approach for supporting mobility ([3] and [4]). The gateway into each domain is called the Domain Root Router (DRR). The Mobile Host (MH) keeps it network address unchanged while moving within a domain. The Corresponding Nodes (CN) and the Home Agent (HA) do not need to be aware of the host's position within the domain. To reach the MH, HAWAII uses specialized path setup schemes that update forwarding entries in specific routers. When a router receives a packet for an unknown $\mathrm{MH}$, it uses a preconfigured default interface pointing towards the domain root router. The packet will be forwarded in that direction till it arrives at a router that knows a route to the $\mathrm{MH}$. 


\subsection{Description of the Handoff Protocol in HAWAII}

There are two kinds of path setup schemes. One for networks with MHs that can only be connected to one base station (e.g. TDMA networks) and the other kind of schemes for MHs that can be simultaneously connected to two or more base stations at the same time (e.g. CDMA networks). From the four schemes considered in [3], we consider only the Multiple Stream Forwarding (MSF). This scheme forwards packets from the Old Base Station (BSO) to the New Base Station (BSN) before being diverted at the crossover router (i.e. a router where the path from $\mathrm{CN}$ to $\mathrm{BSO}$ and the path from $\mathrm{CN}$ to $\mathrm{BSN}$ cross).

In order to describe the operation of the MSF path setup scheme, we define the following messages (see Fig. 2.). At the instant of handoff, BSO loses contact with the $\mathrm{MH}$ and at the same time the $\mathrm{MH}$ sends a MIP registration message (M1) to BSN. The latter sends a path setup update message M2 to the BSO. When M2 arrives at $\mathrm{BSO}$, the BSO starts to forward all packets with destination $\mathrm{MH}$ via router $\mathrm{R} 1$, including those packets that arrive after the handoff instant and that were stored in a forwarding buffer at the BSO. For that purpose, BSO adds a forwarding entry to its routing table indicating that packets for $\mathrm{MH}$ should leave the $\mathrm{BSO}$ via interface $\mathrm{A}$. BSO sends the path setup message (M3) to R1, who adds a forwarding entry to its routing table indicating that packets for the $\mathrm{MH}$ should leave the $\mathrm{R} 1$ via interface $\mathrm{A}$. R1 sends the path setup message (M4) to R0, who adds a forwarding entry indicating that packets for the MH should leave the R0 via interface C. From this instant on, all packets arriving at router R0 are sent directly to BSN. The path setup message continues (M5 and M6) triggering similar actions until it reaches BSN.

\subsection{Performance Evaluation of HAWAII}

The use of the HAWAII MSF path setup scheme implies that packets with destination the $\mathrm{MH}$ will follow a route, depending on their time of arrival at certain routers. In what follows, the packets are divided in classes according to the path they follow. The timing of these classes will be given from the point of view of a packet originating from the corresponding host that arrives at R0. Let $t_{h o}$ be the time instant of handoff (i.e. the time instant that BSO loses contact with the $\mathrm{MH}$ and that $\mathrm{M} 1$ is generated in the $\mathrm{MH}$ ). We assume that the time needed to update the forwarding entries is about equal to the service time of any packet in any router.

Class 1 packets: These packets arrive at the BSO after the handoff took place and before the new forwarding entry is added at the BSO. They arrive at the R0 after

$$
t_{0}=t_{h o}-\{R 0(B)+(R 0, R 1)+R 1(B)+(R 1, B S O)\}
$$

but before

$$
t_{1}=t_{h o}+\{(M H, B S N)+B S N(A)+(B S N, R 2)+R 2(A)+(R 2, R 0)+R 0(B)\}
$$

These packets are stored in the forwarding buffer of the BSO until they are forwarded to the MH using the route R0-R1-BSO-[forwarding buffer]-R1-R0-R2-BSN. 
Class 2 packets: These packets arrive at R1 before M3 causes the adding of the new forwarding entry at R1, and they arrive at the BSO after the new forwarding entry has been added. They reach the $\mathrm{MH}$ via the route R0-R1-BSO-R1-R0-R2-BSN. At time instant

$$
\dot{t_{1}}=t_{1}+(R 0, R 1)+R 1(B)+(R 1, B S O)+B S O(A)+(B S O, R 1)+R 1(A)
$$

message 3 is processed and router R1 changes its forwarding entries for packets with destination the $\mathrm{MH}$. Hence, the packets that arrive at R0 in the interval $\left[t_{1}, t_{2}\right]$, with

$$
t_{2}=t_{1}^{\prime}-\{R 0(B)+(R 0, R 1)\}
$$

belong to class 2 .

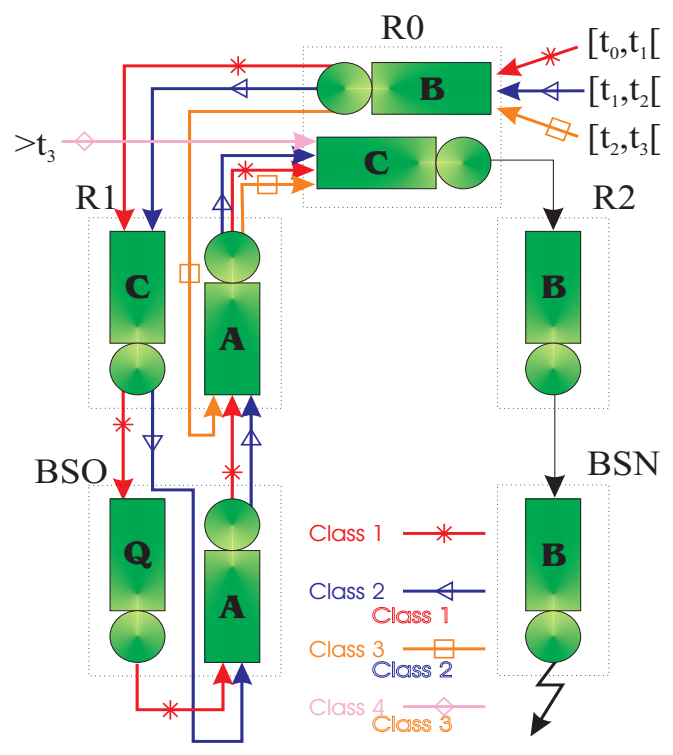

Fig. 3. A queuing model for the MSF scheme

Class 3 packets: These packets arrive at R0 before M4 causes the adding of the new forwarding entry at R0, and they arrive at R1 after the new forwarding entry has been added. They reach the $\mathrm{MH}$ via the route R0-R1-R0-R2-BSN. At time instant

$$
t_{3}=t_{2}+R 0(B)+(R 0, R 1)+(R 1, R 0)+R 0(C)
$$

router R0 changes its forwarding entries for packets with destination the $\mathrm{MH}$ (based on message M4). Therefore, packets arriving at R0 in the interval $\left[t_{2}, t_{3}\right]$, belong to class 3 .

Class 4 packets: These packets are forwarded to the BSN directly, i.e. via the route R0-R2-BSN. They arrive at R0 after time instant $\mathrm{t} 3$. 
Remark: The MSF scheme may result in the creation of routing loops (for example, after BSO has changed its entry to forward packets but before Router 1 processes M3). However these loops exist only for extremely short periods of time. In our model we only consider possible loops for packets that belong to class 1 , as the probability for the occurrence of other types of loops seems to be negligible.

We derive two performance measures for this handoff scheme: the additional delay introduced by the forwarding scheme and the packet loss probability in the forwarding buffer of BSO.

Consider a stream of packets originating from the corresponding host arriving at R0 with a constant interarrival time of $T$ ms. Let the arrival instant $u$ of the first packet at R0 be uniformly distributed over $\left[t_{0}, T\right]$. In what follows we let $t_{0}=0$. Then it is possible to compute the probability distribution of the additional time introduced by the MSF scheme. In fact, this is the time needed for the packet to reach R0 (possibly after being routed via $\mathrm{R} 1$ and $\mathrm{BSO}$ ). Remark that for packets arriving at R0 after time instant $t_{3}$, this additional time is zero (as no forwarding is applied anymore).

The probability that the $k$-th packet of the stream (arriving at R0 at time instant $(k$ 1) $\times T+u$ ) arrives back in R0 later than time instant $(k-1) \times T+u+t$ (i.e. it experiences an extra delay of $t$ due to the forwarding mechanism) is given by the following expression.

$\operatorname{Prob}[k$-th packet arrives at R0 later than instant $(k-1) \times T+t+u]=$

$\operatorname{Prob}\left[(k-1) \times T+u<t_{1}\right]$

$\times \operatorname{Prob}\left[k\right.$-th packet has extra delay larger than $\left.t \mid(k-1) \times T+u<t_{l}\right]$

$+\operatorname{Prob}\left[t_{1} \leq(k-1) \times T+u<t_{2}\right]$

$\times \operatorname{Prob}\left[k\right.$-th packet has extra delay larger than $\left.t \mid t_{1} \leq(k-1) \times T+u<t_{2}\right]$

$+\operatorname{Prob}\left[t_{2} \leq(k-1) \times T+u<t_{3}\right]$

$\times \operatorname{Prob}\left[k\right.$-th packet has extra delay larger than $\left.t \mid t_{2} \leq(k-1) \times T+u<t_{3}\right]$

$+\operatorname{Prob}\left[t_{3} \leq(k-1) \times T+u\right]$

$\times 0$

In the above, the probability expression regarding packets of class 1 can be established as follows. It is assumed that, as soon as the new forwarding entries are set in BSO, the packets of class 1 that are stored in the forwarding buffer, are put in the queue of output interface A. Hence, they will be forwarded to R1, but when they arrive at R1 before the forwarding entries are updated, they will be sent right back to BSO. This looping occurs roughly when the waiting and service of the packet in the output queue take less time than the waiting and processing of M3 in R1. We denote the service rate of a packet $\mu$. Then we can write: 
$\operatorname{Prob}\left[k\right.$-th packet has extra delay larger than $\left.\mathrm{t} \mid(k-1) \times T+u<t_{l}\right]=$ Prob[ $k$-th packet gets in loop]

$$
\begin{aligned}
\times & \operatorname{Prob}[R O(B)+(R 0, R 1)+R 1(B)+(R 1, B S O)+(t 1-(k-1) \times T-u) \\
& +(k-1) / \mu+B S O(A)+(B S O, R 1)+R 1(B)+(R 1, B S O)+B S O(A) \\
& \left.+(B S O, R l)+R 1(A)+(R 1, R O)>t \mid(k-1) \times T+u<t_{1}\right]
\end{aligned}
$$

$+\operatorname{Prob}[k$-th packet does not get in loop]

$$
\begin{aligned}
& \times \operatorname{Prob}[R O(B)+(R O, R 1)+R 1(B)+(R 1, B S O)+(t 1-(k-1) \times T-u) \\
& +(k-1) / \mu+B S O(A)+(B S O, R 1)+R 1(A)+(R 1, R O)>t \mid(k-1) \\
& \left.\times T+u<\mathrm{t}_{1}\right]
\end{aligned}
$$

Clearly, if $k=1$ the packet has a larger probability $(\operatorname{Prob}[B S O(A)<R l(A)])$ of getting into this routing loop than if $k=2(\operatorname{Prob}[B S O(A)+(B S O-1 / \mu)<R 1(A)])$. The looping probability of the possible other class 1 packets is considered to be negligible.

Regarding class 2 packets, we do not consider possible loops, so:

$\operatorname{Prob}\left[k\right.$-th packet has extra delay larger than $\left.t \mid(k-1) \times T+u<t_{2}\right]=$

$$
\begin{gathered}
\operatorname{Prob}[R 0(B)+(R 0, R 1)+R 1(B)+(R 1, B S O)+B S O(A)+(B S O, R 1) \\
\left.+R 1(A)+(R 1, R 0)>t \mid t_{1} \leq(k-1) \times T+u<t_{2}\right] .
\end{gathered}
$$

Finally, for class 3 packets we can write:

$\operatorname{Prob}\left[k\right.$-th packet has extra delay larger than $\left.t \mid t_{2} \leq(k-1) \times T+u<t_{3}\right]=$

$$
\operatorname{Prob}\left[R 0(B)+(R 0, R 1)+R l(A)+(R 1, R 0)>t \mid t_{2} \leq(k-1) \times T+u<t_{3}\right] .
$$

To compute the different delay components we assume that all routers on a path that a packet follows are modelled as simple $\mathrm{M} / \mathrm{M} / 1$ queueing systems. The exponential service time of a packet both includes the time needed for the routing of the packet as well as the time needed to actually put the packet on the output link. We denote the corresponding load of a router $\rho$. Conditioning on the value of $u$, the arrival instant of the first packet of the stream, all probabilities occurring in the expression for the delay packets experience due to the forwarding mechanism are of the form $\operatorname{Prob}[X+c<t]$, where $X$ is a random variable being the sum of response times in an $\mathrm{M} / \mathrm{M} / 1$ queue and $c$ a constant. It is well known that the distribution of the response time in an $\mathrm{M} / \mathrm{M} / 1$ queue is again an exponential distribution with rate $\mu(1-\rho)$. Hence the random $X$ is a sum of exponentially distributed random variables. It is possible to find an explicit expression for the distribution of $X$, and hence the value of $\operatorname{Prob}[X+c<t]$ can be computed explicitly. Also some more complicated probabilities that occur in the expression, such as $\operatorname{Prob}[X+c<t<Y+d], \operatorname{Prob}[X+$ $Y+c \leq t<X+Z+d]$ or $\operatorname{Prob}[X+c<t \mid Y<d]$ ( $X, Y$ and $Z$ sums of exponentially distributed variables), can be computed through some standard conditional probability techniques. Applying these general expressions for the special cases above leads to the probability that the $k$-th packet of the stream arrives at R0 later than instant $(k-1)$ $\times T+u+t$.

Now we evaluate the packet loss in the forwarding buffer in BSO. Clearly the number of packets that need to be buffered is exactly the number of packets belonging to class 1 . Hence, the probability that $k$ packets need to be stored equals 


$$
P\left((k-1) \times T+u \leq t_{1}<k \times T+u\right) .
$$

Using the assumption that the routers are modelled as $\mathrm{M} / \mathrm{M} / 1$ queues, this probability can be computed easily.

We now want to compute the expected number of packets that will be dropped due to the expiration of a playout buffer at the $\mathrm{MH}$ because of the forwarding mechanism introduced by the MSF scheme. A packet will be dropped in case of a playout delay of $t$ whenever it experiences an extra delay (due to forwarding) larger than $t$.

To approximate the expected number of dropped packets, we consider the different classes separately:

$E[$ number of dropped packets $]=$

$\sum_{i=1,2,3} E[$ number of dropped packets of class $i]$.

For $i=2,3$ we have that

$E[$ number of dropped packets of class $i]=E[\#$ class $i$ packets $] \times p_{i}$,

where $p_{i}$ denotes the probability that a class $i$ packet is dropped. This probability is the same for every class $i$ packet and is of the form $\mathrm{P}[X+c>t], X$ being a sum of response times, so $p_{i}$ can be computed.

To obtain the expected number of packets in the respective classes, we consider the following probability distributions:

$\operatorname{Prob}[\#$ class 1 packets $=k]=\operatorname{Prob}\left[(k-1) \times T+u \leq t_{1}<k \times T+u\right]$,

$\operatorname{Prob}[\#$ class 1 and 2 packets $=k]=\operatorname{Prob}\left[(k-1) \times T+u \leq t_{2}<k \times T+u\right]$,

Prob[\# class 1, 2 and 3 packets $=k]=\operatorname{Prob}\left[(k-1) \times T+u \leq t_{3}<k \times T+u\right]$.

These probabilities are of the same form as before and the expected value of the distributions can be easily computed. Furthermore, we have that

$\mathrm{E}[\#$ class 2 packets $]=\mathrm{E}[\#$ class 1 and 2 packets $]-\mathrm{E}[\#$ class 1 packets $]$

$\mathrm{E}[\#$ class 3 packets $]=\mathrm{E}[\#$ class 1,2 and 3 packets $]$ - E[\# class 1 and 2 packets].

Now to obtain an approximation for E[number of dropped packets of class 1], let $n_{I}$ denote the smallest integer larger than $\mathrm{E}$ [\# class 1 packets]. Furthermore, let $p_{I, k}$ be the probability for the $k$-th packet of the stream to be dropped, given that it belongs to class 1 . For every $k$, this probability can be computed as pointed out before. Then, with $p_{l}$ denoting the mean value of $p_{\mathrm{l}, 1}, \mathrm{~K}, p_{\mathrm{l}, n_{1}}$, we have (approximately) :

$E$ [number of dropped packets of class 1]

$$
=n_{1} \times p_{1}-\left(n_{1}-\mathrm{E}[\# \text { class } 1 \text { packet }]\right) \times p_{1, n_{1}} .
$$

This gives an approximation for the expected number of packets dropped in the playout buffer due to the MSF forwarding mechanism. 


\subsection{Numerical Results}

In this section we apply the above analytical models to investigate the following performance measures. First, the packet loss probability as a function of the capacity of the forwarding buffer in BSO is determined. Next the distribution of the delay experienced by packets that arrive after handoff due to the use of the forwarding scheme is computed.

Consider the system depicted in Fig. 1, where each router (including the Base Stations) has the following characteristics: the service rate $\mu$ equals 10 packets per $\mathrm{ms}$, the load is given by $\rho=0.8$, and the propagation delay to each neighbouring router is variable $(5 \mathrm{~ms}, 10 \mathrm{~ms}$ and $20 \mathrm{~ms})$. We consider a stream of packets arriving at Router R0 with a constant packet interarrival time of $T=20 \mathrm{~ms}$.

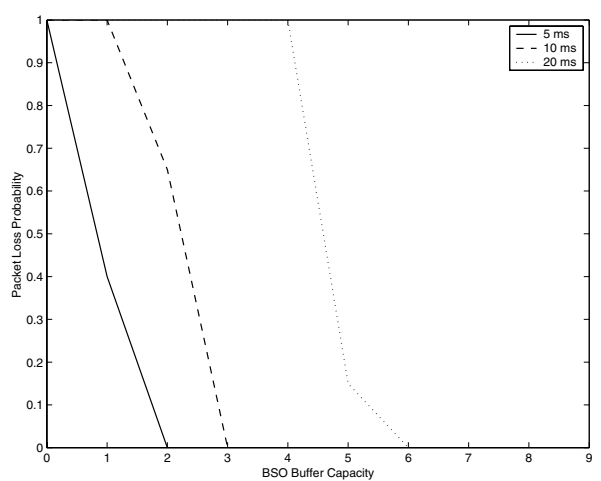

Fig. 4. Packet loss probability in the Forwarding Buffer

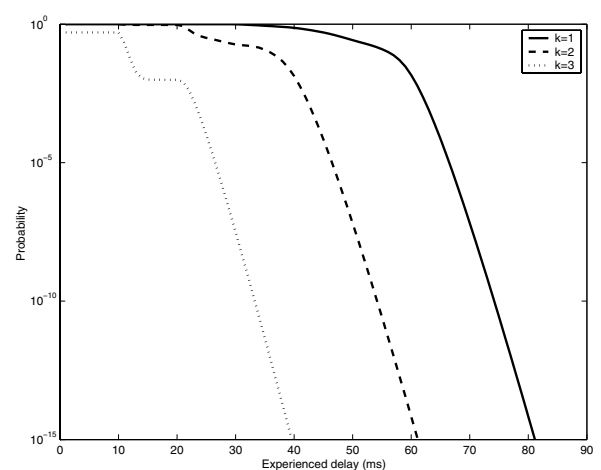

Fig. 5. Delay experienced by packets due to forwarding

For this system, Fig. 4. shows the packet loss probability as a function of the capacity of the forwarding buffer at the Old BS for the different propagation delays between neighbouring routers ( $5 \mathrm{~ms}, 10 \mathrm{~ms}$ and $20 \mathrm{~ms}$ ).

Next, we compute the delay due to forwarding for this system. Fig. 5. shows the probability that the $k$-th packet after handoff experiences a delay of at least $t$ ms due to the forwarding scheme.

The same evaluation is made for different values of the router service time. We assume a system where the propagation delay to the neighbouring routers is $5 \mathrm{~ms}$ and variable router service rate ( 2 packets $/ \mathrm{ms}, 5$ packets $/ \mathrm{ms}$ and 10 packets $/ \mathrm{ms}$ ). Figure 6 shows the delay experienced by the first and second packet for the different values of the service rate $\mu$.

Now we give an example where the expected number of packets that are dropped for a certain playout delay are computed, for variable router service rate $\mu$. We consider again the system described in the previous examples, with the load of each router $\rho=0.8$, the propagation delay to each neighbouring router $5 \mathrm{~ms}$ and variable router service rate $(1$ packet $/ \mathrm{ms}, 2$ packet $/ \mathrm{ms}, 5$ packet $/ \mathrm{ms}$ and 10 packet $/ \mathrm{ms})$. We consider a stream of packets arriving at Router R0 with a constant packet interarrival 
time of $T=20 \mathrm{~ms}$. Fig. 7 shows the expected number of packets dropped as a function of the playout buffer for the different values of $\mu$.

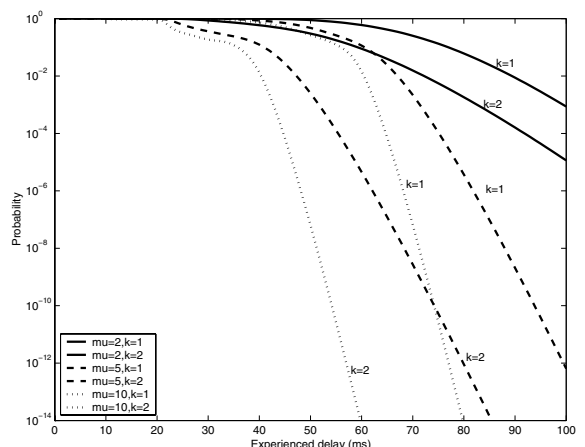

Fig. 6. Delay experienced by packets due to forwarding for variable router service rates.

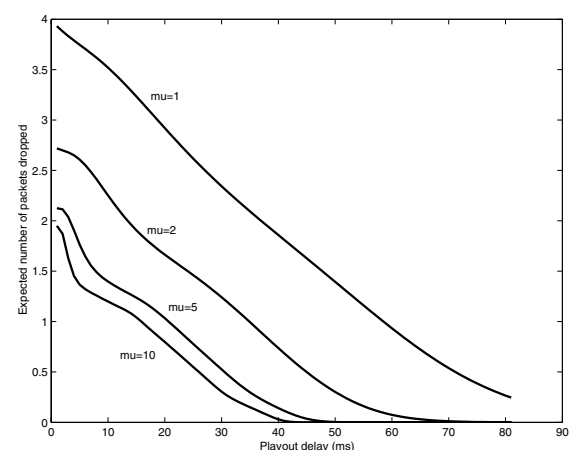

Fig. 7. Expected number of packets dropped due to expiration of playout delay

\section{Cellular IP}

In this Section we briefly describe Cellular IP and present an analytical model to evaluate the packet loss and delay experienced by packets involved in a handoff.

\subsection{Network Model and Routing}

A Cellular IP ([5],[6],[7]) access network is connected to the Internet via a gateway router. A MH attached to an access network will use the IP address of the gateway as its Mobile IP care-of address. The main component of Cellular IP networks is the base station that is used as a wireless access point to which the MH relays the packets it wants to transmit and from which it receives the packets destined to it.

After power up a $\mathrm{MH}$ has to inform the gateway router about its current point of attachment by means of a route update message. This message is received by the base station and forwarded to the gateway on a hop-by-hop basis. Each Cellular IP node maintains a route cache in which it stores host based routing entries. The path taken by uplink packets is cached by all intermediate nodes. To route downlink packets addressed to the $\mathrm{MH}$, the path used by recently transmitted packets from the $\mathrm{MH}$ and which have been cached is reversed.

As the routing entries in the nodes are soft state, a $\mathrm{MH}$ that has no data to transmit, has to send periodically special IP packets to maintain its routing path. Paging is used to route packets to MHs that have not been transmitting or receiving data for a while and whose routing cache entries have timed out. Paging caches are not maintained in each node and have a longer timeout value. If a node without a paging cache, receives a packet for a $\mathrm{MH}$ for which it has no routing cache entry, it will broadcast it to all its downlink neighbours. 


\subsection{Description of the Handoff Protocol in Cellular IP}

In Cellular IP a mobile host initiates a handoff (based on signal strength measurements) by sending a route update packet to the new base station. This packet travels in a hop-by-hop manner from the base station to the gateway router and reconfigures the route cache entries in the Cellular IP nodes along its way.

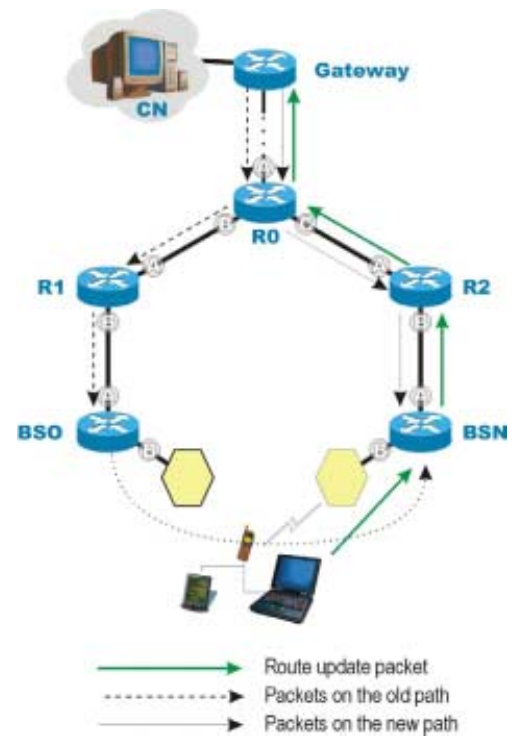

Fig. 8. Handoff in Cellular IP

Cellular IP supports three types of handoff mechanisms: hard handoff, semi-soft handoff and indirect semi-soft handoff.

1. Hard Handoff: The wireless interface of a mobile host changes from one base station to another at once. Data packets arriving at the cross-over node before the route cache entry is changed are misdirected and will be lost since the mobile host is already attached to the new base station.

2. Semi-soft Handoff: The mobile host switches to the new base station, transmits a route update packet with a flag indicating a semi-soft handoff while listening to the old base station. The route update packet reconfigures the route caches on the way to the gateway router and adds an entry to the cache of the crossover node. Downlink packets for the mobile host are duplicated in the crossover node and sent along both paths, the new one and the old one. After a semi-soft delay, the mobile host performs a regular handoff. It migrates to the new base station and sends another route update packet to complete the handoff. This last packet stops the crossover node duplicating packets. The semi-soft delay is a fixed amount of time that is proportional to the mobile-to-gateway round-trip delay. If the path to the new base station is shorter than to the old one, some packets may not reach the mobile host. To overcome this problem, packets sent along the new path need to be delayed. A delay device mechanism, located at the crossover node, 
should provide sufficient delay to compensate for the time difference between the packets travelling on the old and new paths.

3. Indirect Semi-soft Handoff: This mechanism can be applied when using wireless technologies that have no simultaneous connection capability. They cannot listen to the old base station while sending a route update packet to the new base station. To initiate a handoff the mobile host sends the route update packet to the old base station, using as destination IP address the address of the new base station. The old base station forwards this packet with a flag indicating indirect semi-soft handoff uplink to the gateway. The gateway delivers the packet to the new base station using normal IP routing. When the new base station receives this packet, it creates a semi-soft route update packet with the IP address of the mobile host and it forwards it upstream. The rest of the algorithm then works as a semi-soft handoff.

\subsection{Performance Evaluation of Cellular IP}

We evaluate two performance measures for Cellular IP, namely the number of packets lost in the Hard Handoff scheme and the delay that has to be introduced in R0 to compensate for the difference in the old and new paths in the Semi-soft Handoff scheme.

First we remark that the number of packets lost in the BSO during Hard Handoff equals the number of packets that have to be stored in BSO in the MSF scheme of HAWAII. Therefore we can use the model described in Section 3.3.

In order to compute the delay that has to be introduced in R0 to compensate for the difference in old and new paths in the Semi-soft Handoff scheme, we first give an expression of the length of these paths, $L_{\text {old }}$ and $L_{\text {new }}$ respectively.

$$
\begin{aligned}
& L_{\text {old }}=R 0(B)+(R 0, R 1)+R 1(B)+(R 1, B S O)+B S O(B) \\
& \text { Lnew }=R 0(C)+(R 0, R 2)+R 2(B)+(R 1, B S N)+B S N(B)
\end{aligned}
$$

Let again the arrival instant $u$ of the first packet at R0 be uniformly distributed over $\left[t_{0}, T\right]$. Then the probability that the number of lost packets during handoff exceeds $\mathrm{k}$ is given by

$$
\begin{aligned}
& \mathrm{P}(\# \text { lost packets during handoff } \geq k) \\
& \quad=P\left(L_{\text {old }}-u>L_{n e w}+(N+k-1) \times T\right)
\end{aligned}
$$

Since all service times in the routers are exponentially distributed, this probability can be computed easily.

\subsection{Numerical Results}

First we evaluate the case of the Hard Handoff scheme. Consider the reference network (Fig. 1.), where the service rate $\mu$ of each router equals 10 packets per ms, the load is given by $\rho=0.8$, and the propagation delay to each neighbouring router is 
variable ( $5 \mathrm{~ms}, 10 \mathrm{~ms}$ and $20 \mathrm{~ms}$ ). We consider a stream of packets arriving at Router R0 with a constant packet interarrival time, taking values between $5 \mathrm{~ms}$ and $35 \mathrm{~ms}$. Fig. 9. shows the expected number of packets that are lost at the BSO due to the hard handoff, for variable packet interarrival times and different values of propagation delay. Clearly the expected number of lost packets increases when the propagation delay increases and the interarrival time decreases. Remark that the results obtained in Fig.9. correspond with those obtained in the evaluation of the number of buffers required in BSO in the HAWAII protocol.

Next, for the Semi-soft Handoff scheme we compute the packet loss probability as a function of the number of packets that are delayed in the R0 buffer before the first packet is released, in order to cope with the different propagation delay between R0BSO and R0-BSN. We assume this difference to be $50 \mathrm{~ms}, 75 \mathrm{~ms}$ and $100 \mathrm{~ms}$. The three curves in Fig. 10 show the loss probability of packets due to early arrival in BSN for different values of the buffer capacity in R0.

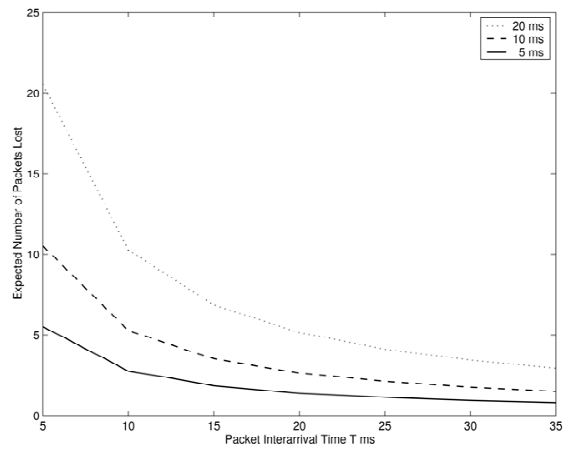

Fig. 9. Hard handoff : Expected number of packets lost in BSO

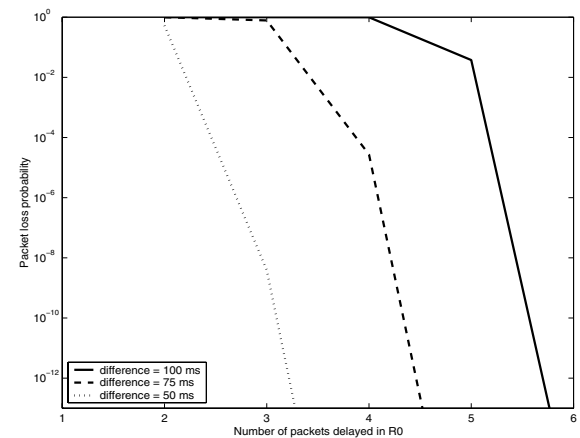

Fig. 10. Packet loss probability as a function of number of delayed packets for different values of difference in length of new and old path

\section{Comparison HAWAII - Cellular IP}

In this Section we use the models developed in Sections 3 and 4to compare performance aspects of the MSF scheme of HAWAII and the Semi-soft Handoff scheme of Cellular IP. In particular, we compare the number of buffers that are required in both schemes to assure a zero or negligible packet loss and we compare the delay induced by the use of these handoff schemes. Notice that this comparison is not completely fair as the mobile host's capabilities are not the same. For the Semisoft handoff scheme in Cellular IP, the MH must be able to send a route update packet to the new BS, while listening to the old BS, whereas in the MSF scheme the MH switches instantaneously from the old BS to the new BS.

The MSF scheme of HAWAII requires buffers in order to store packets that arrive in BSO after the handoff occurs and before the forwarding entry in BSO is updated, i.e. to store temporarily class 1 packets. In Section 3 we have shown how this number 
of buffers can be obtained. The R0 buffers required in the Cellular IP have a different task. They are required to delay packets before sending them along the new path in order to compensate for the time difference between the old and the new path to the $\mathrm{MH}$. A model that allows to evaluate this required number of buffers has been proposed in 3.3 .

Consider the system presented in Fig. 1., where the routers have a service rate $\mu$ of 10 packets $/ \mathrm{ms}$, the load of each router is given by $\rho=0.8$, the distance between neighbouring nodes on the old route (i.e. on the route R0-R1-BSO-MH) is $20 \mathrm{~ms}$ and the distance between the neighbouring nodes on the new route is variable, taking values $1 \mathrm{~ms}, 3 \mathrm{~ms}, 5 \mathrm{~ms}, 10 \mathrm{~ms}$ and $15 \mathrm{~ms}$. We consider a stream of packets arriving at Router R0 with a constant interarrival time of $T=20 \mathrm{~ms}$. Fig.11. compares the number of buffers required in BSO when using the HAWAII MSF scheme with the number of buffers required in R0 when using the Semi-soft handoff scheme of Cellular IP, in order to obtain a zero packets loss. This result shows that Cellular IP requires less buffers than HAWAII. This is due to the fact that in Cellular IP the buffers are needed to compensate for the time difference between the old path and the new path, while in HAWAII, the buffers need to accommodate packets arriving during a time equal to the sum of the old path and the new path (i.e. the time required by M2 to reach BSO). Clearly, the longer the new path, the more buffers HAWAII requires. Cellular IP on the other hand requires less buffers since a longer new path leads to less difference between the new and old path.

Next we compare the delay introduced by both schemes. Consider again the same system as defined in the above example. We use the models derived in II.B and III.B to obtain this delay. For the HAWAII MSF scheme we compute for each inter-router distance, the expected delay the $k$-th $(k=1,2,3, .$.$) packet experiences. We compute this$ delay for values of $k$ for which this delay is not negligible (i.e. for all packets that are involved in the handoff). By taking the average of these values, we obtain the average delay a general packet, that is involved in the handoff, experiences. Remark that delays for different values of $\mathrm{k}$ may be quite different; e.g. when the distance between neighbouring routers on the new path is $10 \mathrm{~ms}$, then the delay the first packet (i.e. $k=1$ ) experiences is $165 \mathrm{~ms}$, while the delay for the eighth packet (i.e. $k=8$ ) is $30 \mathrm{~ms}$.

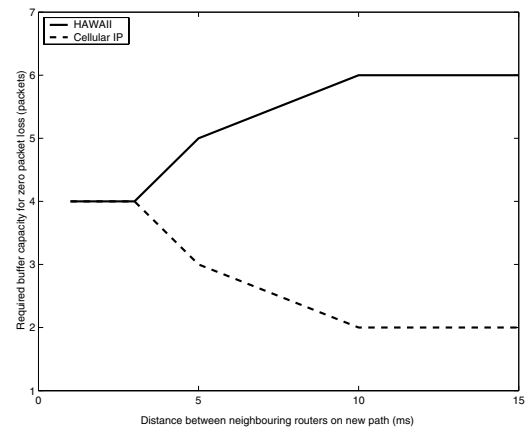

Fig. 11. Comparison of the buffers required in MSF HAWAII and Semisoft Handoff in Cellular IP

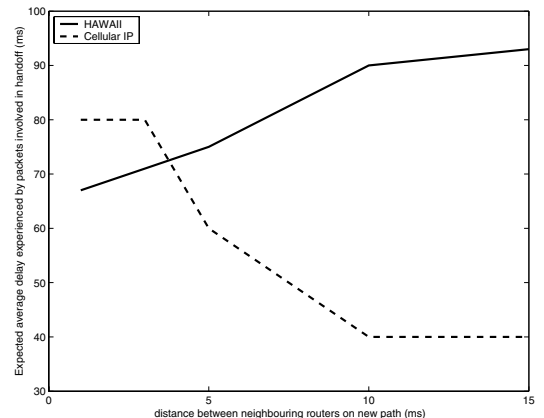

Fig. 12. Comparison of the delay experienced by packets involved in the handoff in MSF HAWAII and Semi-soft Handoff in Cellular IP 
From Fig. 12., we see that in this case the average delay is given by $90 \mathrm{~ms}$. For the Cellular IP scheme, the delay experienced by every packet that is involved in the handoff equals the number of buffers obtained in Fig.10. multiplied by the packet interarrival time $T=20 \mathrm{~ms}$. Fig. 12. compares the results for the two schemes. This figure shows that, apart from the case of very small distances between neighbouring nodes, packets in Cellular IP will experience (in average) less delay than packets in HAWAII. Remark however that in this numerical example, we have assumed that the difference in length (in ms) between the old and new path is known. In a real environment, this difference is not known and a conservative (worst case) value has to be selected, leading to possible much longer delays. In HAWAII, not all packets experience the same delay. The first packets may have a much longer delay than the last packets that are involved in the handoff. This phenomenon, which is not clear from Fig. 12., is illustrated in Fig. 5.

\section{Conclusions}

We have developed simple analytic models to evaluate the performance of handoff schemes of two important micro-mobility solutions, namely HAWAII and Cellular IP. The models that are proposed are simple $\mathrm{M} / \mathrm{M} / 1$ queueing networks that incorporate propagation delays between routers and processing times within routers. The models are not developed for dimensioning purposes, but mainly to investigate the influence of important design parameters and to compare the two solutions. For this reason we have assumed Poisson background traffic and exponential processing times. A detailed validation of the approximations has been made in [8] by comparison with simulation. The simplicity of the model also allows the study of more general network topologies than the one considered in this paper.

The performance analysis mainly focuses on packet loss probabilities during handoff and extra packet delay introduced by the handoff schemes. For the HAWAII MSF scheme we compute the required capacity of the buffer in the old Base Station in order to have zero or negligible packet loss. Moreover we compute the delay distribution experienced by the different packets involved in the handoff. This delay can be used to compute the number of lost packets when a playout buffer is used at the destination. For the Cellular IP protocol, we derive the number of dropped packets when using a hard-handoff scheme. Moreover, for the semi-soft handoff scheme, we compute the number of packets that have to be delayed before releasing the first packet in order to compensate for the time difference between the old path and the new path.

These results are used to make a comparison of the MSF scheme in HAWAII and the semi-soft handoff scheme in Cellular IP. It is shown that, in general Cellular IP needs less buffers than HAWAII, mainly due to the fact that in Cellular IP the buffers are needed to compensate for the time difference between the old path and the new path, while in HAWAII, the buffers need to accommodate packets arriving during a time interval equal to the sum of the old path and the new path. With respect to the delay introduced during handoff, we have seen that the delay is constant for all 
packets involved in the semi-soft handoff scheme of Cellular IP. But as the difference in length between the old path and the new path is not known in advance, the delay that is enforced in the cross-over router may be difficult to engineer. In the MSF scheme in HAWAII on the contrary, the delay a packet experiences is variable and decreases with the sequence number of the packet involved in the handoff. This extra delay is a result of the forwarding scheme itself and does not need to be engineered.

\section{References}

1. C. Perkins, ed., "IP Mobility Support”, IETF RFC 2002, October 1996.

2. R. Caceres and V. Padmanabhan, "Fast and scalable handoffs for wireless networks", in Proc. ACM MOBICOM '96, pp. 56-66, 1996

3. Ramjee, R., La Porta, T., Thuel, S., Varadhan, K., and Wang, S., HAWAII: a domain based approach for supporting mobility in wide-area wireless networks, Proceedings of International Conference on Network Protocols, ICNP'99.

4. R. Ramjee, T. La-Porta, S. Thuel, K. Varadhan, L. Salgarelli, "IP micro-mobility support using HAWAII", Internet draft, July 2000.

5. A. Valko, "Cellular IP - a new approach of Internet host mobility", ACM Computer Communication Reviews, January 1999

6. A. Campbell, J. Gomez, C. Y Wan, S. Kim, Z. Turanyi, A. Valko, "Cellular IP", IETF draft (draft-ietf-mobileip-cellularip-00.txt), January 2000.

7. A. Campbell, J. Gomez, S. Kim, A. Valko, C.-Y. Wan and Z. Turanyi, "Design, implementation and evaluation of Cellular IP", IEEE Personal Communications, August 2000, pp.42-49

8. C. Blondia, O. Casals, L. Cerda and G. Willems, "Performance analysis of a forwarding scheme for handoff in HAWAII", to appear in Proceeedings of Networking 2002, Pisa 2002, win-www.uia.ac.be/u/pats/publications.html 